\title{
Exploring liver physiology, pathology, TGF- $\beta$, EMT, stemness and new developments in liver cancer
}

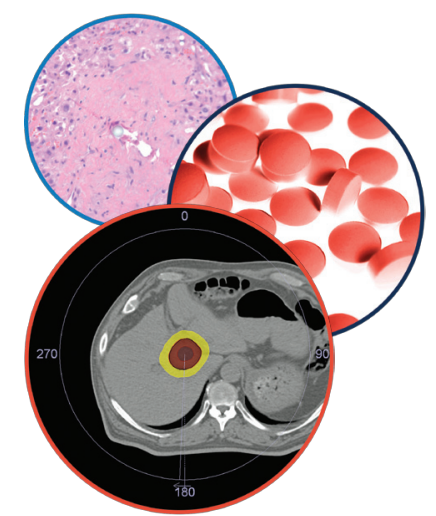

Hepatic Oncology

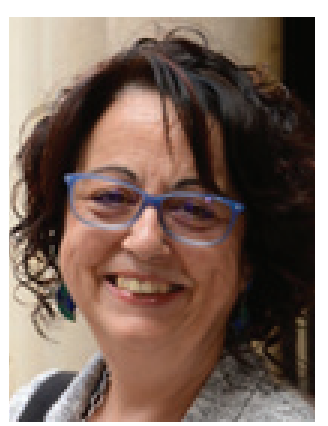

Isabel Fabregat* speaks to Roshaine Wijayatunga, Managing Commissioning Editor. Isabel Fabregat, $\mathrm{PhD}$, is Senior Investigator, Head of the group "TGF- $\beta$ and cancer", at the Bellvitge Biomedical Research Institute (IDIBELL) and Associate Professor in the School of Medicine and Health Sciences at the University of Barcelona. She is the President of the Spanish Society for Cell Biology since 2011, she was member of the Executive Board of the Spanish Branch of the European Tissue Culture Society and she is currently member of the Spanish Society for the Study of the Liver, and the International Liver Cancer Association, among others. The current research line of her laboratory focuses on the dissection of the molecular mechanisms induced by TGF- $\beta$ in liver cells that can explain its different, and sometimes opposite, functions in liver pathologies, in particular fibrosis and hepatocarcinogenesis. Cross-talk between TGF- $\beta$ and other pathways, in particular EGFR signaling, is also one of the current interests of the lab. From October 2012 she has coordinated an Initial Training Network - Marie Curie European Action that under the acronym of 'IT-LIVER' (Inhibiting TGF- $\beta$ in Liver Diseases) integrates different European academic groups and small enterprises to develop a multidisciplinary training program for talented young researchers, to prepare them for leading roles in chronic liver disease research. Dr Fabregat has published around 130 articles in indexed journals, as well as several book chapters, with her work published in Hepatology, Journal of Hepatology, Genes \& Development, Cancer Research, Oncogene, FASEB Journal, Free Radical in Biology \& Medicine, Journal of Cell Science, Journal of Biological Chemistry, among others. She is an editorial member of the World Journal of Gastroenterology, World Journal of Hepatology, Frontiers in Physiology-Gastrointestinal Sciences, Frontiers in Pharmacology, Current Signal Transduction Therapy and Hepatic Oncology.

First draft submitted: 24 March 2017; Accepted for publication: 29 March 2017; Published online: 6 July 2017

\section{Q When did you become interested in liver physiology \& pathology, in particular what prompted you to begin your research on TGF- $\beta$, epithelial-mesenchymal transition \& stemness in cancer?}

My interest in the liver started during my predoctoral at the Autónoma University in Madrid, in the beginning of 1980s, where I studied regulation of the oxidative metabolism in isolated hepatocytes from rats. During my first postdoc in the Complutense 
University in Madrid, I continued working with hepatocytes, now in primary culture, which offered enormous advantages for long-term experiments. During these two periods I started to become fascinated by the complexity of the liver, the cross-talk of pathways that are required for its correct function, as well as the wonderful process of liver regeneration.

My interest in TGF- $\beta$ ? It is a little longer story. During my postdoc, at the Imperial Cancer Research Foundation (ICRF) in London, I became enthused by signal transduction mechanisms. Upon my return to Madrid, when I had the opportunity to start my independent research line, I decided to explore new signals regulating hepatocyte functions and TGF- $\beta$ was introduced into my life! From the beginning, it was clear that it could mediate several functions, activating different signaling pathways. As time progressed, stronger evidence suggested its involvement in liver pathologies.

TGF- $\beta$ is one of the stronger inducers of epithelial-mesenchymal transition (EMT), and I have an excellent colleague and friend working in this field, Dr Ángela Nieto. Indeed, with her help I did an immersion in the area of cell migration and invasion linked to the EMT process. A sabbatical year at UCSD, in La Jolla, San Diego, at the end of the 1990s, made me think about how little was known in the area of liver stem cells and, in particular, their potential role in liver cancer. So this is the reason why I decided to explore the role of EMT on hepatocyte differentiation and stemness.

In 2005 I moved to the Bellvitge Biomedical Research Institute, in Barcelona, and focused my research on liver pathologies, particularly HCC, in collaboration with clinicians from the Hospital de Bellvitge. From then, my group has been focused on trying to better understand the role of the TGF- $\beta$ pathway in liver carcinogenesis, its cross-talk with other pathways, such as EGF, and its relevance in modulating EMT and stemness. During the last years, we have been working in the framework of a European consortium coordinated by our group from Barcelona: IT-LIVER.

\section{Q Could you briefly describe the relationship} between EMT \& stemness in liver cancer?

Different evidences initially suggested that TGF- $\beta$ induces an EMT process in nontumoral epithelial cells, which correlated with the acquisition of a less differentiated phenotype. In fact, it was suggested that it was the activation of the EMT program that conferred properties of stem cells (for a review, see [1]). In this sense, in collaboration with Dr Nelson Fausto, we found that when human fetal hepatocytes are chronically treated with TGF- $\beta$, they undergo EMT, coincident with downregulation of specific hepatic genes and upregulation of stem cell markers, reminiscent of progenitor-like cells. These mesenchymal stem-like cells redifferentiated to either hepatocytes or bile duct cells after undergoing mesenchymal-epithelial transition (MET) in response to controlled experimental conditions, which was concomitant with the reexpression of liver-specific genes. Different evidences later suggested that TGF- $\beta$ may give rise to tumor-initiating cells in liver cirrhosis and cancer. However, there is still controversy in the ways in which EMT and stemness are connected, as well as whether or not this connection is relevant for metastasis. In fact, a number of groups have recently reported that MET is required for efficient metastatic colonization and that EMT may be not necessarily associated with stemness [1]. In fact, cancer cells undergo dynamic and reversible transitions, switching from a fully differentiated epithelial to a dedifferentiated mesenchymal state, but passing for multiple intermediate situations. In this sense, we have recently described that TGF- $\beta$ may induce a partial EMT in some epithelial hepatocellular carcinoma (HCC) cells, increasing the expression of stem-related mesenchymal genes (such as CD44), but maintaining epithelial gene expression. The most interesting finding was that these mixed epithelial-mesenchymal cells show higher stemness potential than the mesenchymal ones. Worthy to note that in HCC patient samples, TGFB1 expression most frequently correlates with a partial EMT, that is, increase in stem-related mesenchymal genes, but maintenance or overexpression of the epithelial ones. So, there are no black or white situations, this is cell biology! No doubt that further work is necessary to completely understand the crosstalk between EMT and stemness in the context of liver cancer.

Q EMT \& stemness are believed to contribute to resistance to chemotherapy, with a role for miRNAs. Is there a potential for epigenetic drugs to reverse resistance to therapy?

Yes, of course, EMT and stemness, either separately or coordinately, might confer resistance to chemotherapeutic drugs in cancer cells. It is very well known that the tumor initiating cells 
contribute to chemoresistance. Due to the implication of the EMT process not only in promoting cell motility and invasion but also in the generation of a cancer stem (tumor initiating) cell phenotype, both EMT and stemness may contribute to drug resistance. Expression of some stem genes, such as CD133 or CD44 confers chemoresistance in different types of cancer, including HCC. CD44 is a characteristic mesenchymal stem-related gene. Different evidences from our and other groups have indicated that CD44 induced by TGF- $\beta$ would mediate both EMT and stemness [1]. Interestingly, the combination of a mesenchymal phenotype and expression of CD44 impairs the response of HCC cells to sorafenibinduced apoptosis. Corroborating this, dedifferentiated stem-like HCC-derived cell lines presented enhanced expression of stem-related genes, such as $C D 44$, and showed resistance to anticancer drugs. Furthermore, a recent study in HCC xenografts in mice has revealed that acquired resistance to sorafenib is driven by tumor initiating cells with enrichment of progenitor markers.

There is no doubt that miRNAs are controlling EMT and stemness and, indeed, chemoresistance. Different miRNAs have been associated to the EMT phenotype by regulating the expression of EMT-related transcription factors [1]. Unfortunately, we do not have much information in the case of the HCC, although some recent evidences indicate that down-regulation of some miRNAs in liver tumor cells may provoke phenotypic changes correlating with EMT and chemoresistance. However, it would be very simplistic to think that a miRNA only mediates its action through this process. The miRNAs act on mRNAs from many different genes. And recent reports suggest that alteration of miRNA expression in HCC could mediate chemoresistance by acting on other processes, such as autophagy or metabolism. Whether EMT, autophagy and metabolism are connected in the framework of $\mathrm{HCC}$ is a challenge for next investigations and will reveal new aspects to explain drug resistance.

Epigenetics is a whole in itself. Not only by its potential implication in chemotherapy resistance, but also because it may contribute to the initiation, progression and dissemination of HCC. I consider that there is an excellent future for epigenetic drugs, alone or in combination with other drugs. In particular, histone deacetylase (HDAC) inhibitors have been successful in clinical trials in HCC, in combination with sorafenib. Whether these inhibitors may contribute to change the EMT and stemness phenotype of the HCC cells is matter of study in different laboratories, including ours.

\section{Q Along similar lines is there potential for the} inhibition of transcription factors, for example Snail1, as a therapeutic target?

No right now, this is much more complicated. As mentioned above, we need to better understand how the metastasis process occurs and the involvement of EMT in the process. Of course inhibitors of EMT-related genes could potentially block the EMT, but they could also contribute to potentiate the metastasis at late stages, where the epithelial phenotype needs to be recovered and MET must occur. However, we cannot lose the perspective for the use of Snail1 inhibitors in other pathological processes, such as fibrosis, where EMT would play a role. This is another controversial issue in the liver, but for other tissues, such as kidney, it is very clear that EMT contributes to the initiation and expansion of the myofibroblasts.

Q How important will the development of new animal models be to the study of these pathogenic processes in liver cancer?

Very important, of course. The cell biology studies, in vitro, are insufficient for understanding the organism as a whole. Animal models are very helpful for reproducing human pathologies, as well as for understanding the role of a specific gene in these pathologies. Even though a lot of studies have revealed the different intracellular pathways that contribute to adult liver regeneration, the specific timing of these events is not fully understood, neither the overlapping among them. Hepatocarcinogenesis is a long process, with a sequence of alterations and genetic or epigenetic mutations, a multifactorial process. Without doubt, the animal models help us to better understanding these sequential events. In this sense, we have recent results in a new animal model generated by our group, in collaboration with some colleagues in the Complutense University and the CIEMAT in Madrid, indicating that the EGFR pathway in hepatocytes is essential only in the early stages of hepatocarcinogenesis in mice and, unexpected, it mainly contributes to the inflammatory process. Once the tumor is initiated, the EGFR is not essential and may be replaced by other signaling pathways. This would explain the low success of EGFR inhibitors in clinical trials for advanced HCC. 
Q The process of initiation \& metastasis of cancer is very complex. Do you think we will ever understand enough to treat or even prevent liver cancer?

I am positive. A narrow connection between basic and clinical research is necessary for improving our knowledge about the molecular mechanisms that provoke the initiation of a liver carcinogenic process and to understand the mechanisms that provoke its dissemination. The throughput analyses with omic technologies are helping a lot, as well as the new animal models generated in different labs. We not only need the development of new drugs, but also the design of biomarkers that may anticipate the success of a specific treatment in each patient. A great effort for the collaboration among surgeons, oncologists, pathologists and basic researchers has been done in the last years and, hopefully, will be translated in great advances in the close future.

Q Could you briefly describe your work with the IT-LIVER Consortium on the role of TGF- $\beta$ in liver disease?

IT-LIVER was born with the aim of joining efforts in Europe to better understand the role of the TGF- $\beta$ pathway in liver chronic diseases, mainly fibrosis and cancer [2]. Different academic and company groups have been collaborating during the last years to clarify whether TGF- $\beta$ may be a targetable pathway in liver diseases $[3,4]$. We were granted by a FP7 Marie Curie - Initial Training Network program. Indeed, we did not only focus our efforts in research, but also in training. This kind of Initial Training Network actions has the advantage that they are multidisciplinary programs, with basic and clinical groups, and participation of the industry. This creates an excellent environment for the training of a new generation of scientists in this area of research, which were incorporated in our groups as pre- or post-doctoral. What is worthy of comment is that our program has impacted on the formation of external young investigators too, since from IT-LIVER we have organized different courses and international conferences, opened for free to external participants. It has been a very rewarding experience.

\section{Q The IT-LIVER Consortium has a focus of} training for young researchers. How important is it for established researchers to encourage $\&$ train the new generation of scientists in this field?
Very important! It is part of our responsibility, the training of future generations for the new era of the 21st century science. But, furthermore, the creation of the consortium has allowed different groups, which previously were competitors, to become collaborators and friends. All the $\mathrm{PhD}$ or postdoc programs have been performed with the participation of, at least, two different IT-LIVER partners. The fellows have made secondments in other academic or company labs. All these interactions have been very positive not only for the fellows, but also for the groups.

Q What direction will your research be taking over the next few years?

In our group we have focused our current research on better understanding molecular processes that are regulated by TGF- $\beta$ and that can be relevant in HCC. The final aim is to enhance our knowledge for the establishment of potential biomarkers that may help for stratifying patients according to a 'TGF- $\beta$ signature' that could anticipate the success of anti-TGF- $\beta$ therapies. We have recently opened new avenues of study and new technologies. Transcriptomics, proteomics and metabolomics are helping us to discover new functions for TGF- $\beta$, or for its downstream signals that were unknown. We have given special attention to a protein: the NADPH oxidase NOX4, which mediates the suppressor effects of TGF- $\beta$ and whose expression and/or function would be inhibited in HCC patients. We have incorporated to our work a new animal model NOX4 null where we are studying liver regeneration and hepatocarcinogenesis. And we are moving toward metabolism as a relevant process modulated by TGF- $\beta$ in HCC, concomitant with the EMT/stemness process.

Financial \& competing interests disclosure The author has no relevant affiliations or financial involvement with any organization or entity with a financial interest in or financial conflict with the subject matter or materials discussed in the manuscript. This includes employment, consultancies, honoraria, stock ownership or options, expert testimony, grants or patents received or pending, or royalties.

No writing assistance was utilized in the production of this manuscript.

\section{Disclaimer}

The opinions expressed in this interview are those of the interviewee and do not necessarily reflect the views of Future Medicine Ltd. 
Exploring liver physiology, pathology, TGF- $\beta$, EMT, stemness \& new developments in liver cancer

\section{References}

1 Fabregat I, Malfettone A, Soukupova J. New insights into the crossroads between EMT and stemness in the context of cancer. J. Clin. Med. 5(3), pii:E37 (2016).
2 IT-Liver. http://www.it-liver.eu/

3 Giannelli G, Mikulits W, Dooley $S$ et al. The rationale for targeting TGF- $\beta$ in chronic liver diseases. Eur. J. Clin. Invest. 46(4), 349-361 (2016).
4 Fabregat I, Moreno-Càceres J, Sánchez A et al. IT-LIVER Consortium. TGF- $\beta$ signalling and liver disease. FEBS J. 283(12), 2219-2232 (2016). 